of NSAIDs is characterized by lower pain intensity than as against colchicine or glucocorticoids.

Disclosure of Interests: Maria Chikina: None declared, Maxim Eliseev Speakers bureau: Novartis, Menarini Group, Alium

DOI: 10.1136/annrheumdis-2020-eular.5164

\section{AB0922 1 BUROSUMAB (ANTI-FGF23 MONOCLONAL ANTIBODY) IN THE TREATMENT OF A PATIENT WITH RECURRENT TUMOR INDUCED OSTEOMALACIA.}

C. Crotti ${ }^{1}$, F. Zucchi ${ }^{1}$, P. Messa ${ }^{2}$, R. Caporali ${ }^{1,3}$, M. Varenna ${ }^{1} .{ }^{1}$ Gaetano Pini Institute, Dept of Rheumatology, Milan, Italy; ${ }^{2}$ Unit of Adult Nephrology, Dialysis and Renal Transplant, Foundation Ca' Granda IRCCS Ospedale Maggiore Policlinico, Dept of Medicine, Milan, Italy; ${ }^{3}$ University of Milan, Dept of Clinical Sciences and Community Health, Milan, Italy

Background: Tumor-induced Osteomalacia (TIO) is a rare paraneoplastic syndrome caused by tumoral overproduction of fibroblast growth factor 23 (FGF23) resulting in hyperphospaturia, hypophosphatemia and osteomalacia. Surgery is the only curative treatment, but tumor can locally recur, even after years from primary surgery. Furthermore, some tumors cannot be removed by surgery due to their location.

Objectives: To describe a case of a 53-year-old woman affected by recurrent $\mathrm{TIO}$ after three surgical attempts of removal treated with Burosumab.

Methods: We describe the case of a 53 years old woman with TIO treated with Burosumab, an anti-FGF-23 monoclonal antibody at present approved for X-linked hypophosphatemic rickets only.

Results: A 46-year-old Caucasian female was referred to our Bone Unit after experiencing several fractures in different sites. She reported being in good health until three years prior consultation. At the time of symptoms onset, she experienced a progressive muscle pain, enabling her to stand for a long period. During imaging evaluation for atraumatic fracture of right great trochanter, the MRI abdomen and ${ }^{18}$ FDG- PET-CT showed a metabolic pre-sacral lesion. She unsuccessfully underwent to an exploratory laparotomy of that lesion. Then, she suffered from atraumatic intertrochanteric fracture of right femur, surgically treated, and after 3 months, she had an insufficiency dyaphiseal fracture of the left femur, surgically treated. Furthermore, she experienced several ribs fractures. At the time of first evaluation, lab works showed: serum-Phosophate (PS) $1.2 \mathrm{mg} / \mathrm{dL}$ (reference range (RR) $2.5-4.5 \mathrm{mg} / \mathrm{dL}$ ), urinary-phosphate of $24 \mathrm{~h}$ (PU) $842 \mathrm{mg} / 24 \mathrm{~h}$, alkaline phosphatase (ALP) $565 \mathrm{UI}(\mathrm{RR}<300), 1,25(\mathrm{OH})$ vitamin D $27 \mathrm{ng} / \mathrm{L}$ (RR 25-86.5), PTH 24 (RR<75 pg/mL), intact-FGF-23 117 (RR 25-45 pg/mL), normal serum and 24h-urinary Calcium. Patient underwent to ${ }^{68} \mathrm{Ga}$-DOTATATE-PET-CT that showed a pre-sacral lesion, who was studied with MRI and CT before surgery. In 2013 patient underwent surgical excision of the pre-sacral region. After 18 months of well-being, patient complained worsening of articular pain and muscle weakness, and further ribs fractures. Another ${ }^{68} \mathrm{Ga}$-DOTATATE-PET-CT reported a relapse of the previous pre-sacral lesion $(32 \times 12 \times 47 \mathrm{~mm})$ with an increase of FGF-23levels $(54.6 \mathrm{pg} / \mathrm{mL})$. Even the subsequent surgery was not able to remove the tumor. Since 2015, patient was maintained in phosphorus supplements and $1,25(\mathrm{OH})_{2}$ vitamin $\mathrm{D}_{3}$, but PS levels never normalized. We asked for compassionate use of Burosumab and, after ethical committee approval, in September 2019 she was started on Burosumab, at a dose of $30 \mathrm{mg}$ per month $(0.3 \mathrm{mg} / \mathrm{kg})$. At baseline, she had PS $1.2 \mathrm{mg} / \mathrm{dL}$, PU $1874 \mathrm{mg} / 24 \mathrm{~h}$, TRP $25.96 \%$. After two months, she improved in pain symptom (VAS reduction from 65 to $12 \mathrm{~mm}$ ), which allow her to walk and stand without crutches. She did not normalize her PS levels $(1.3 \mathrm{mg} / \mathrm{dL})$, while PU reduced to $1000 \mathrm{mg} / 24 \mathrm{~h}$. We titred Burosumab dose at $40 \mathrm{mg}$ per month $(0.6 \mathrm{mg} / \mathrm{kg})$ and patient is still under therapy, waiting for next blood works.

Conclusion: This is the first European patient affected by TIO treated with Burosumab. Burosumab could be a promising therapy in the medical treatment of TIO refractory or not eligible for definitive surgery. Further data are needed to standardize the proper dose regimen.

Disclosure of Interests: Chiara Crotti: None declared, Francesca Zucchi: None declared, Piergiorgio Messa: None declared, Roberto Caporali Consultant of: AbbVie; Gilead Sciences, Inc.; Lilly; Merck Sharp \& Dohme; Celgene; Bristol-Myers Squibb; Pfizer; UCB, Speakers bureau: Abbvie; Bristol-Myers Squibb; Celgene; Lilly; Gilead Sciences, Inc; MSD; Pfizer; Roche; UCB, Massimo Varenna: None declared

DOI: 10.1136/annrheumdis-2020-eular.4466

\section{AB0923 DIETARY FACTORS AND TOPHI: A FOOD INTAKE FREQUENCY SURVEY IN CHINESE GOUT PATIENTS}

Q. H. LI ${ }^{1}$, Y. F. Bi ${ }^{1}$, X. H. Lin ${ }^{2}$, H. B. Wang ${ }^{3}$, C. Y. Wu ${ }^{3}$, C. Deng ${ }^{1}$, J. D. MA ${ }^{1}$, H. W. Lu', J. J. Liang ${ }^{1}$, L. Dai ${ }^{1}$. 'Sun Yat-Sen Memorial Hospital, Sun Yat-Sen University, Department of Rheumatology, Guangzhou, China; ${ }^{2}$ Sun Yat-Sen Memorial Hospital, Sun Yat-Sen University, Department of Clinical Nutrition,
Guangzhou, China; ${ }^{3}$ Zhongshan School of Medicine, Sun Yat-Sen University, Guangzhou, China

Background: Tophi is a cardinal sign of advanced gout. Risk factors of gout are also closely related to the formation of tophi, such as impaired kidney function and serum uric acid (sUA). Several dietary factors, such as alcohol, fructose-containing beverage, red meat, sea foods have been confirmed increasing the risk of gout. Diet patterns vary widely in different countries. Dietary factors' association with tophi formation remain elusive in Chinese gout patients.

Objectives: This study aimed to study whether dietary factors were risk factors for tophi.

Methods: We recruited consecutive gout patients who fulfilled the 2015 Gout Classification Criteria of ACR/EULAR and collected demographic data, gout disease characteristics and comorbidities. Tophi was evaluated by physical examination and/or musculoskeletal ultrasound. All gout patients completed 10-items food intake frequency questionnaire which included red meat, animal offal, seafood, alcohol, fructose-containing beverages, milk and dairy products, coffee, hotpot slow-cooking soup and tea. Patients were advised to report the average frequency of food consumption in the preceding year of first gout attack. Multivariate logistic regression analysis was performed to evaluate risk factors of tophi. Dependent variables were those met $p$ values less than 0.1 on univariate analysis.

Results: a)There were 682 gout patients recruited with $94 \%$ male, mean age $44 \pm 16$ years, and median gout duration $4(2,7)$ years. The mean sUA was $9.0 \pm 2.3 \mathrm{mg} / \mathrm{dl}$. Tophi presented in $166(24.3 \%)$ patients with $31(4.5 \%)$ patients diagnosed by ultrasound. In patients with gout duration $<3$ years, 3 4.9 years, $5 \sim 9.9$ years and $\geq 10$ years, the prevalence of tophi were $6.7 \%, 19.4 \%, 38.8 \%$, and $49.6 \%$, respectively. b)Tophus patients were characterized by older age $(48 \pm 16$ vs. $42 \pm 15$ years), longer gout duration $[7(4,10)$ vs. $3(1,5)$ years], more ever involved joints $[11(4,24)$ vs. $3(2,5)]$ and more flare times in the last year $[11(4,24)$ vs. $3(2,6)]$. For comorbidities, tophus patients presented higher prevalence of urolithiasis (36\% vs. $23 \%$ ), hypertension ( $54 \%$ vs. $40 \%$,) and diabetes ( $20 \%$ vs. $11 \%$ ) but less hypertriglyceridemia ( $19 \%$ vs. $32 \%$, all $\mathrm{P}<0.05$ ). c)Compared with patients without tophi, tophus gout patients consumed more red meat (>300g/d: $12 \%$ vs. $6 \%$ ), seafood (>2 times/w: $18 \%$ vs. $13 \%$ ), hotpot ( $\geq 1$ time/w: $17 \%$ vs. $10 \%$ ) and alcohol (>84g/d: $23 \%$ vs. $9 \%$ ). d)Dependent variables of multivariate logistic regression analysis included age, gender, gout duration, diuretics, BMI, sUA, serum creatinine, urine $\mathrm{pH}$, hypertriglyceridemia, hypertension, diabetes, coronary heart disease, urolithiasis, alcohol consumption, hotpot, red meat, and seafoods. Gout duration, sUA, serum creatinine and urine $\mathrm{pH}$ were positively correlated with tophi, while hypertriglyceridemia was negatively associated with tophi. For dietary factors, heavy alcohol consumption (> 84g/ day vs. $<1 \mathrm{~g} /$ day $\mathrm{OR}=2.624,95 \% \mathrm{Cl}: 1.437-4.793$ ) and hotpot $(\geq 1$ time/w vs. $<1$ time/w, OR=2.164 $95 \% \mathrm{Cl}: 1.217-3.847)$ were positively correlated with tophi.

Conclusion: Our data suggest tophi should not be ignored in gout patients with short duration. Heavy alcohol consumption and hotpot are associated with the formation of tophi.

Disclosure of Interests: None declared

DOI: 10.1136/annrheumdis-2020-eular.4007

\section{AB0924 CALCIFIC TENDONITIS OF THE ROTATOR CUFF: PERIOSTIN ENRICHMENT IS ASSOCIATED WITH A BETTER RESPONSE TO ULTRASOUND-GUIDED PERCUTANEOUS LAVAGE.}

C. Darrieutort-Laffite ${ }^{1,2}$, P. Arnolfo, ${ }^{1,2}$, E. Correia ${ }^{1}$, F. Blanchard ${ }^{1}$, B. Le Goff ${ }^{1,2}$ ${ }^{1}$ INSERM U1238, Nantes, France; ${ }^{2}$ Nantes University Hospital, Rheumatology, Nantes, France

Background: Calcific tendonitis of the rotator cuff is a frequent cause of chronic shoulder pain. It is due to apatite deposits within the tendons. Little data are currently available about proteins associated to crystals within deposits.

Objectives: The aim of the study was to quantify 6 proteins in calcific powders obtained from patients who have undergone an ultrasound-guided percutaneous lavage (UGPL) of their calcification and to look for correlations between their concentration and patient characteristics.

Methods: Calcific powders were obtained from patients included in the CALCECHO trial whose main objective was to compare post-procedure pain between two groups: methylprednisolone or placebo injected at the end of the lavage [1]. Based on preliminary proteomic data and literature data, the following proteins have been selected and quantified by ELISA: Pigment-epithelium Derived Factor (PEDF), Osteopontin (OPN), Periostin (POSTN), Activin A (ACT A), Osteoprotegerin (OPG) and Bone Morphogenic Protein-2 (BMP-2). The level of each protein was expressed in $\mu \mathrm{g}$ per pg of the total proteins present in the sample. These proteins have been selected for their link to the mineralization. Correlations between the level of each protein and radiographic and ultrasound appearance of the calcific deposits were sought. We also looked for correlations between leve of each protein and duration of pain or response to UGPL (Mann-Whitney test). 